\title{
Polarization Properties of Stimulated Brillouin Scattering and their Implications on Slow and Fast Light
}

\author{
Moshe Tur ${ }^{1}$, Avi Zadok ${ }^{2}$, Elad Zilka ${ }^{1}$, Avishay Eyal ${ }^{1}$, and Luc Thévenaz ${ }^{3}$ \\ Invited Paper \\ ${ }^{1}$ School of Electrical Engineering, Faculty of Engineering, Tel-Aviv University, Tel-Aviv 69978, Israel \\ ${ }^{2}$ Department of Applied Physics, MC 128-95, California Institute of Technology, Pasadena, CA 91125, USA \\ ${ }^{3}$ Ecole Polytechnique Fédérale de Lausanne, Institute of Electrical Engineering, STI-GR-SCI Station 11, CH-1015 Lausanne, Switzerland \\ tur@eng.tau.ac.il avizadok@caltech.edu avishay@eng.tau.ac.il luc.thevenaz@epfl.ch
}

\begin{abstract}
Vector formalism for stimulated Brillouin scattering amplification in birefringent fibers is used to model the fiber as an equivalent, pseudo-linear, polarization-dependent gain medium. Implications on slow and fast light setups are discussed.

(C)2009 Optical Society of America

OCIS codes: (290.5900) Scattering, stimulated Brillouin; (120.5410) Polarimetry; (060.2300) Fiber measurements.
\end{abstract}

\section{Introduction}

Stimulated Brillouin scattering (SBS) is a non-linear optical propagation effect, in which a high power pump wave and a typically weaker, counter-propagating signal wave are coupled by a longitudinal acoustic wave [1]. Given that proper phase matching requirements are met, the signal power may be exponentially amplified (Stokes wave), or attenuated (anti-Stokes wave). The amplification, or attenuation, of the signal is accompanied by frequency-varying phase delays, in accord with the Kramers-Kronig relations [1]. Within a limited bandwidth, these phase variations are a nearly-linear function of frequency, representing an additive positive or negative group delay for the Stokes and anti-Stokes waves, respectively. These group delays, easily controlled by the pump power, have made SBS a favorable underlying mechanism in many studies of slow and fast light [2-3]. Many efforts have been dedicated over the last four years to broadening the usable bandwidth of SBS slow light, and to reducing the distortion of the delayed signals [4-8]. Both high rate digital data [7-9], and broadband radar signals [10], were successfully delayed.

Since SBS originates from optical interference, the SBS interaction, at a given point, is most efficient when the electric fields of the pump and signal are aligned, i.e., their vectors trace parallel ellipses and in the same sense of rotation. Conversely, if the two ellipses are again similar, but traced in opposite senses of rotation, with their long axes being orthogonal to each other, then the SBS interaction averages to zero over an optical period. Consequently, the overall signal gain depends on fiber birefringence, as well as on the input states of polarization (SOPs) of both waves. In this work, we provide vector formalism for SBS interaction in birefringent fibers, in both Jones and Stokes spaces [11]. The analysis shows that in the undepleted pump regime, the fiber may be regarded as an equivalent, pseudo linear, polarization dependent gain medium, characterized by a pair of orthogonal input SOPs which give rise to the maximum and minimum output signal power. Using these two axes as a convenient basis, we predict that the output SOP corresponding to an arbitrarily polarized input signal is drawn towards a specific state, which is determined by the input pump SOP. This prediction is supported by both simulations and experiments [11]. A similar effect was recently studied in stimulated Raman amplification [12].

The polarization properties of SBS have significant implications on slow and fast light realizations. As the signal gain depends on its input polarization, so does the delay, and polarization control and tracking is often required. This difficulty was recently alleviated by the introduction of a Faraday rotating mirror (FRM) in a doublepass configuration [13]. In addition, an arbitrarily polarized signal could lead to non-linear dependence of the SBS induced delay on the pump power [14]. Finally, the projection of a signal pulse to the maximum and minimum gain axes may also lead to SBS-induced polarization mode dispersion (PMD) and considerable distortion, as the two components experience different group delays.

\section{Vector formalism for SBS in birefringent fibers}

Let us denote the Jones column vectors of monochromatic signal and pump waves as $\vec{E}_{\text {sig }}(z), \vec{E}_{\text {pump }}(z)$, with $z$ the position along a fiber of length $L$. We assume that the difference between the two optical frequencies equals the Brillouin shift $v_{B}$, for maximum interaction [1]. We restrict the analysis to the undepleted pump regime and neglect linear fiber losses. Birefringence is represented by the Jones matrix $\mathbf{T}(z)$. The frequency dependence of $\mathbf{T}(z)$ is 
neglected, since $v_{B}$ is only $\sim 10 \mathrm{GHz}$ and $L$ is only a few $\mathrm{km}$. Subject to the assumptions above, the equations of propagation for the signal and pump waves are given by [1, 15-16]:

$$
\begin{gathered}
\frac{\mathrm{d} \vec{E}_{\text {sig }}(z)}{\mathrm{d} z}=\left[\frac{\mathrm{d} \mathbf{T}(z)}{\mathrm{d} z} \mathbf{T}^{\dagger}(z)+\frac{\gamma_{0}}{2} \vec{E}_{\text {pump }}(z) \vec{E}_{\text {pump }}^{\dagger}(z)\right] \vec{E}_{\text {sig }}(z) \Rightarrow \vec{E}_{\text {sig }}(z)=\mathbf{H}(z) \vec{E}_{\text {sig }}(0), \\
\vec{E}_{\text {pump }}(0)=\mathbf{T}^{T}(z) \vec{E}_{\text {pump }}(z) \rightarrow \vec{E}_{\text {pump }}(z)=\mathbf{T}^{*}(z) \vec{E}_{\text {pump }}(0),
\end{gathered}
$$

with $\gamma_{0}$ denoting the SBS gain coefficient in $[\mathrm{W} \cdot \mathrm{m}]^{-1}$. Note that the differential equation for the signal wave is linear. The linear matrix $\mathbf{H}(z)$ depends on the fiber birefringence and the input SOPs of both waves, and is generally non-unitary. Nonetheless, using the singular value decomposition technique, $\mathbf{H}(z)$ can be represented as:

$$
\mathbf{H}(z)=\mathbf{U}(z) \cdot\left[\begin{array}{cc}
G_{\max }(z) & 0 \\
0 & G_{\min }(z)
\end{array}\right] \cdot \mathbf{V}^{\dagger}(z),
$$

where $\mathbf{U}, \mathbf{V}$ are unitary matrices and $G_{\max }, G_{\min }$ are the maximum and minimum SBS signal amplitude gains, respectively. Equation (2) states that a birefringent, SBS amplifying fiber is equivalent to a linear, polarization dependent gain medium. The orthogonal signal input SOPs leading to $G_{\max }, G_{\min }$ are $\vec{E}_{\text {sig }}^{\text {in } \max }=\mathbf{V} \cdot\left[\begin{array}{ll}1 & 0\end{array}\right]^{T}$ and $\vec{E}_{\text {sig }}^{\text {in }_{\text {min }}}=\mathbf{V} \cdot\left[\begin{array}{ll}0 & 1\end{array}\right]^{T}$, respectively, and the corresponding output SOPs are $\vec{E}_{\text {sig }}^{\text {out } \max }=\mathbf{U} \cdot\left[\begin{array}{ll}1 & 0\end{array}\right]^{T}, \vec{E}_{\text {sig }}^{\text {out } \min }=\mathbf{U} \cdot\left[\begin{array}{ll}0 & 1\end{array}\right]^{T}$. An arbitrarily polarized input signal may be expressed as $\vec{E}_{s i g}^{i n}=\alpha_{0} \vec{E}_{s i g}^{i n_{\text {max }}}+\beta_{0} \vec{E}_{s i g}^{i i_{\text {min }}}$, leading to the following Jones vector and power for the output signal:

$$
\vec{E}_{\text {sig }}^{\text {out }}=\alpha_{0} G_{\text {max }} \vec{E}_{\text {sig }}^{\text {out } \max }+\beta_{0} G_{\text {min }} \vec{E}_{\text {sig }}^{\text {out_min }} ; P_{\text {sig }}^{\text {out }}=\left|\alpha_{0}\right|^{2}\left|G_{\text {max }}\right|^{2}+\left|\beta_{0}\right|^{2}\left|G_{\text {min }}\right|^{2}
$$

Transforming Eq. (1) to Stokes space, we obtain the following equation for the signal power, and its solution:

$$
\mathrm{d} P_{\text {sig }}(\mathrm{z}) / \mathrm{d} z=\frac{1}{2} \gamma_{0} P_{\text {pump }}\left[1+\hat{s}_{\text {pump }}(z) \cdot \hat{s}_{\text {sig }}(\mathrm{z})\right] P_{\text {sig }}(\mathrm{z}) ; P_{\text {sig }}^{\text {out }}=P_{\text {sig }}(0) \exp \left\{\frac{1}{2} \gamma_{0} P_{\text {pump }} L\left\lfloor 1+\left\langle\hat{s}_{\text {pump }}(z) \cdot \hat{s}_{\text {sig }}(\mathrm{z})\right\rangle_{L}\right]\right\}
$$

In Eq. (4) $P_{\text {sig }}(z)$ denotes the signal power, $P_{\text {pump }}$ is the fixed pump power, $\hat{s}_{\text {pump }}(z)$ and $\hat{s}_{\text {sig }}(z)$ are the unit threeelement Stokes column vectors of the pump and signal waves and \langle\rangle$_{L}$ denotes averaging over $z \in\left[\begin{array}{ll}0 & L\end{array}\right]$. If the fiber birefringence is sufficiently large and the fiber long enough, the pump and signal SOPs along the fiber become evenly distributed on the Poincare sphere. In that limit, it has been shown that the maximum and minimum values of $\left\langle\hat{s}_{\text {pump }}(z) \cdot \hat{s}_{\text {sig }}(z)\right\rangle_{L}$ are $\pm \frac{1}{3}$ [17], with $\vec{E}_{\text {sig }}^{\text {out max }}$ aligned with the complex conjugate of $\vec{E}_{\text {pump }}(L)$ [11]. We therefore find:

$$
G_{\max }=\exp \left(\frac{2}{3} \gamma_{0} P_{\text {pump }} L / 2\right), G_{\min }=\exp \left(\frac{1}{3} \gamma_{0} P_{\text {pump }} L / 2\right)
$$

This result, derived using a non-formal argument, was stated in the pioneering work of van Deventer and Boot [18]. Equation (5) states that for a sufficiently long fiber and/or a sufficiently strong pump, $G_{\max }>G_{\min }$. Equations (3) therefore suggests that unless $\alpha_{0}$ is negligible, $\vec{E}_{\text {sig }}^{\text {out }}$ would be closely aligned with $\vec{E}_{\text {sig }}^{\text {outmax }}$. In addition, the equations suggest that the logarithmic SBS gain for an arbitrarily polarized input signal is not necessarily a linear function of pump power.

Figure 1 shows experimental validation of the above predictions [11]. Panel 1(a) shows the measured SBS signal gain, in logarithmic scale, with the input SOP adjusted for $\vec{E}_{s i g}^{i n_{\text {max }}}$ and $\vec{E}_{\text {sig }}^{\text {in }_{\text {min }}}$. The ratio between the slopes

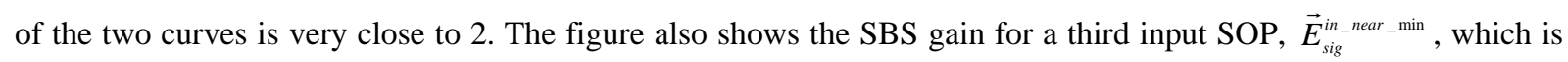
slightly detuned from $\vec{E}_{s i g}^{\text {in_min }}$. The observed logarithmic gain for $\vec{E}_{\text {sig }}^{\text {in_near } \_ \text {min }}$ agrees very well with equation (3), and is not linearly proportional to $P_{\text {pump }}$. The convergence of the output SOP is illustrated in Fig. 1(b). The output SOPs $\vec{E}_{\text {sig }}^{\text {out } \max }$ and $\vec{E}_{\text {sig }}^{\text {out min }}$ are pump power invariant, and orthogonal to each other. The output SOP observed for $\vec{E}_{\text {sig }}^{\text {in_near_min }}$ is gradually drawn from $\vec{E}_{\text {sig }}^{\text {out min }}$ towards $\vec{E}_{\text {sig }}^{\text {out max }}$ as $P_{\text {pump }}$ is increased. 
(a)

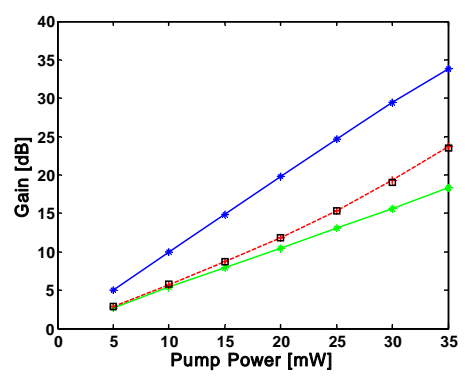

(b)

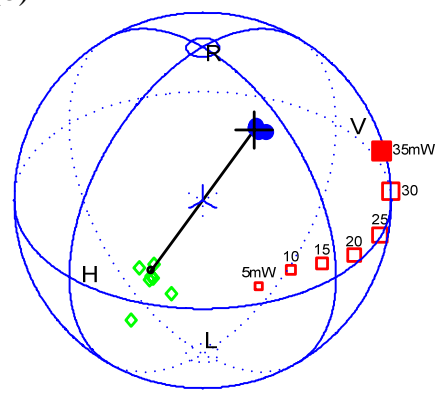

(c)

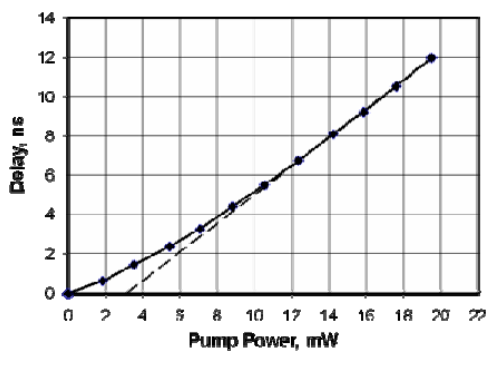

Fig. 1: (a) Measured SBS gain as a function of pump power for a $2250 \mathrm{~m}$ long fiber [11]. The input signal SOP was optimized for maximum output (upper, blue), adjusted for minimum output (lower, green), and fixed at a specific state

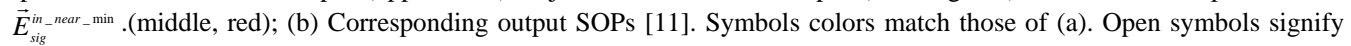
SOPs at the back of the Poincare sphere. Power levels next to the red squares indicate the pump power. (c) Measurement of SBS slow light delay as a function of pump power level [14], showing deviations from a linear relation.

\section{Implications on SBS slow and fast light}

In slow and fast light applications, the monochromatic signal is replaced by a pulse envelope, which may be decomposed in the basis of $\vec{E}_{s i g}^{\text {in } \max }$ and $\vec{E}_{s i g}^{\text {in } \min }$. Each projection would experience a different SBS gain, and hence, as described by the Krmaers-Kronig relation, a different delay as well. Consequently, the observed delay becomes polarization dependent, in accord with the everyday experience of researchers in the field. Following the gain, the delay can also become a non-linear function of $P_{\text {pump }}$, as seen in Fig. 1(c) [14]. $\vec{E}_{\text {sig }}^{i n_{\text {max }} \text { ax }}$ and $\vec{E}_{s i g}^{\text {in }_{\text {min }}}$ may also be regarded as principal axes of an SBS-induced PMD. As the difference between the delays associated with $\vec{E}_{s i g}^{i n_{\text {max }}}$ and $\vec{E}_{\text {sig }}^{\text {in min }}$ can be close to the pulse duration, the distortion associated with such SBS-induced PMD may become severe. This polarization dependence makes SBS slow and fast light setups susceptible to environmental instabilities.

An elegant solution to the polarization sensitivity of SBS slow light was recently proposed by Walker et al. [13], who launched linearly polarized, orthogonal pump and signal waves from the same end of the fiber, with an FRM at the opposite end. In this configuration, the signal wave is guaranteed to interact with a counter-propagating pump wave of a complex conjugate Jones vector, regardless of changes to $\mathbf{T}(z)$ along the fiber. This method is in agreement with the above analysis, which shows that conjugate pump and probe polarizations would provide the maximum SBS gain and delay in a sufficiently long, standard fiber. This result holds for high pump powers [11].

\section{References}

[1] R. W. Boyd, Chapter 9 in Nonlinear optics, pp. 409-427. San Diego, CA: Academic Press, (2003).

[2] K. Y. Song et. al, "Observation of pulse delaying and advancement in optical fibers using stimulated Brillouin scattering," Opt. Epxress 13, 82-88 (2005)

[3] Y. Okawachi et. al, "Tunable all-optical delays via Brillouin slow light in optical fiber, ” Phys. Rev. Lett. 94, 153902 (2005).

[4] M. D. Stenner et. al, "Distortion management in slow light pulse delay," Opt. Express 13, 9995-10002 (2005).

[5] M. González-Herráez et al., “Arbitrary-bandwidth Brillouin slow light in optical fibers,” Opt. Express 14, 1395-1400 (2006).

[6] Z. Zhu et al., "Broadband SBS slow light in an optical fiber,” J. Lightwave Technol. 25, 201-206, (2007).

[7] A. Zadok et al., "Extended delay of broadband signals in stimulated Brillouin scattering slow light using synthesized pump chirp," Opt. Express 14, 8498-8505, (2006).

[8] E. Cabrera-Granado et al., "Observation of large $10 \mathrm{~Gb} / \mathrm{s}$ SBS slow light delay with low distortion using an optimized gain profile," Opt. Express 16, 16032-16042 (2008).

[9] B. Zhang et al., "Slow light on Gbit/s differential-phase-shift-keying signals," Opt. Express 15, 1878-1883 (2007).

[10] A. Zadok et al., "Optically controlled low distortion delay of GHz-wide RF signals using slow light in fibers,” IEEE Photon. Technol. Lett. 19, 462-464 (2007).

[11] A. Zadok et al, "Vector analysis of stimulated Brillouin scattering amplification in standard single-mode fibers,” Opt. Express 16, 2169221707 (2008).

[12] M. Martinelli et al., “Evidence of Raman-induced polarization pulling,” Opt. Express 17, 947-955 (2009).

[13] D. Walker et al., "Stabilizing slow light delay in stimulated Brillouin scattering using a Faraday rotator mirror,” J. Opt. Soc. Am. B 25, C61-C64 (2008).

[14] S. Chin et al., "Zero-gain slow and fast light propagation in an optical fiber,” Opt. Express 14, 10684-10692, (2006).

[15] R. H. Stolen, "Polarization effects in fiber Raman and Brillouin lasers,” IEEE J. Quantum Electron. 15, 1157-1160 (1979).

[16] J. P. Gordon and H. Kogelnik, “PMD fundamentals: polarization mode dispersion in optical fibers,” P. Natl. Acad. Sci. USA 97, 4541-4550, (2000).

[17] F. Corsi et al., “Analytical treatment of polarization mode dispersion in single mode fibers by means of the backscattered signal,” J. Opt. Soc. Am. A 16, 574-583, (1999).

[18] M. O. van Deventer and A. J. Boot, “Polarization properties of stimulated Brillouin scattering in single mode fibers,” J. Lightwave Technol. 12, 585-590, (1994). 